Classification

Physics Abstracts

$61.44+\mathrm{p}$

\title{
Diffuse Scattering in the Icosahedral Al-Li-Cu Quasicrystal
}

\author{
A. Proult $\left({ }^{1}\right)$, P. Donnadieu $\left({ }^{1}\right)$, K. Wang $\left({ }^{2}\right)$ and P. Garoche $\left({ }^{2}\right)$ \\ ( $\left.{ }^{1}\right)$ Laboratoire d'Etude des Microstructures(*). BP 72, 92322 Châtillon Cedex, France \\ $\left({ }^{2}\right)$ Laboratoire de Physıque des Solıdes, CNRS /Université Paris XI, 91405 Orsay Cedex, France \\ (Recelved 11 May 1995, accepted in final form 5 September 1995)
}

\begin{abstract}
Résumé. - Les diagrammes de diffraction électronique des quasicristaux icosaédriques présentent fréquemment des diffusions diffuses. Nous les analysons ici en détails sur des échantillons de phase quasicristalline $\mathrm{Al}_{6} \mathrm{Li}_{3} \mathrm{Cu}$ (T2) traités thermiquement dans lesquels les diffusions diffuses sont très prononcées. Les intensités diffuses forment des batônnets centrés sur des positions appartenant aux rangées réciproques d'ordre 5 et d'ordre 2 et allongés perpendicularrement à ces directions. On montre qu'il s'agit en fait de disques diffus, dans le réseau récıproque à 3 dimensions, dont les positions ne peuvent s'indexer que sur le réseau à 6 dimensions. Toutefois, les intensités ne correspondent pas à celles prédites par l'algorithme de Coupe-et-Projection. Les disques de diffusion diffuse semblent relever d'une organisation quasicristallıne originale se superposant à la phase icosaédrique.
\end{abstract}

\begin{abstract}
Electron diffraction patterns of icosahedral quasıcrystals frequently exhibit diffuse scattermg features. We report a detailed analysis of diffuse scattering in $\mathrm{Al}_{6} \mathrm{Li}_{3} \mathrm{Cu}$ (T2) quasicrystallıne samples. The samples have been specifically heat-treated which allows to observe pronounced diffuse effects. Diffuse streaks are observed along the 5-fold and 2-fold symmetry axes and are elongated perpendicularly to these directions. These streaks are due to discs in the 3-dimensional reciprocal space. The diffuse disc positions are only indexable in the 6-dimensional hyperspace but the disc intensities do not agree with the ones predicted by the Cut-and-Project method. The diffuse discs we observed seem to be related to an original quasicrystalline phenomenon overlapping with the icosahedral phase.
\end{abstract}

Diffuse scattering gives interesting information regarding basic concepts of the quasicrystalline state. On the one hand, the amount of diffuse scattering close to the diffraction peaks is expected to reveal whether quasicrystals are stabilized by disorder or not [1]. On the other hand, the so-called modulated phases, found in the vicinity of the Al-Cu-Fe icosahedral phase, exhibits diffuse scattering and satellites related to precursor states in the quasicrystal/approximant crystal transformation [2]. Hence, either due to modulated phases or random tiling, diffuse scattering appears as a major issue in quasicrystal structure studies.

Basically, the disordered models such as random tiling or icosahedral glass predicts a noticeable amount of diffuse background intensity. In particular, the random tiling predicts diffuse

(*) CNRS/ONERA-UMR 104. 
wings at the Bragg peaks [1]. But, quasicrystal diffraction patterns frequently exhibit localrzed diffuse scattering. Diffuse rings have been observed on precession photographs of Al-Li-Cu cosahedral phase, the so-called T2 phase $[3,4]$. Strong arcs of diffuse scattering on electron diffraction patterns have also been identified in other quasicrystalline phases: $\mathrm{Al}-\mathrm{Mn}, \mathrm{Ti}-\mathrm{Cr}-\mathrm{Si}$, $\mathrm{Al}_{87} \mathrm{Cr}_{17}$. Ti-Mn [5-7]. Actually, it has been shown that these diffuse rings can be accounted for by random tiling [8] and by icosahedral glass models [9]. However, diffuse scattering with other shapes than rings was found by several authors $\mathrm{Al}-\mathrm{Li}-\mathrm{Cu}$ icosahedral phase. But. to our knowledge, these streaks have been only mentioned and briefly commented by Staiger et al. [10]. In other works the diffuse scattering remained unnoticed though clearly visible on the published diffraction patterns $[11,12]$. The detailed analysis of these diffuse streaks, reported here, reveals features never observed with quasicrystal diffuse rings.

The present work aims first at a full description of the diffuse scattering in the $\mathrm{Al}-\mathrm{Li}-\mathrm{Cu}$ icosahedral phase. It is worthwhile to note that diffuse scattering is specifically strong in heat-treated samples, the same diffuse scattering effects, but less pronounced, exist in as-cast $\mathrm{Al}-\mathrm{Li}-\mathrm{Cu}$ quasicrystals. We give a possible interpretation involving microstructural features in the 3-dimensional physical space. However our observation suggests that the six-dimensional crystallography should provide an adequate description.

\section{Diffuse Scattering Streaks on the Electron Diffraction Patterns}

1.1. Experimental Conditions. - We have carred out diffraction studies on $\mathrm{Al}_{6} \mathrm{Li}_{3} \mathrm{Cu}(\mathrm{T} 2)$ cosahedral phase by transmission electron microscopy (TEM) on as-cast and heat-treated samples. The as-cast samples were provided by the Péchiney Research Center and were identical to the icosahedral T2-phase earlier reported by Sainfort and Dubost [13]. The heat-treated samples were obtained using a two-step procedure performed on the as-cast T2 phase samples. The first one was a 2-hours annealing at $615^{\circ} \mathrm{C}$ that allows to eliminate the eutectic phases, the second one was a slow solidification at a $5{ }^{\circ} \mathrm{C} /$ hour cooling rate. The samples were always sealed in a lithium confining device [14].

Slices cut from the bulk material were mechanically polished down to a thickness below 100 microns. Subsequently, the samples were electropolished in a double-jet electropolishing device $\left(T=-35^{\circ} \mathrm{C}\right.$, electrolyte: $1 / 3$ nitric acid. $2 / 3$ methanol). TEM observations were made on a CM20 Philips operating at $200 \mathrm{keV}$ and on a Zeiss EM912 transmission electron microscope operating at $120 \mathrm{keV}$. The Zeiss EM 912 is equipped with an energy filter that allows to remove the noise due to inelastically scattered electrons. Electron Diffraction Patterns (EDP) were taken in the selected area mode. To observe the diffuse scattering. long exposure must be performed (exposure time: 20 to $100 \mathrm{~s}$ ), the condensor lenses being fully defocussed.

1.2. Diffuse Scattering Analysis. - The EDPs displayed in Figure 1 have been taken on a heat-treated Al-Li-Cu icosahedral sample. Streaks of diffuse scattering are obvious on all EDPs (2-fold, 3-fold, 5-fold), some of them are indicated by arrows in Figure 1. Though they are strong close to some intense diffraction spots, the streaks are present in many places of the diffraction pattern. This point is clear in Figure 1c which shows a filtered diffraction pattern taken with a microscope allowing to remove the noise due to ineslastically scattered electrons. The streaks are centered on positions on the 5-fold and 2-fold axes (Figs. 1a, b, c). No diffuse scattering has been observed along the 3 -fold reciprocal row. The streaks are always elongated perpendicularly to the respective reciprocal row.

Two types of Al-Li-Cu quasicrystal were studied: as-cast and heat-treated samples. Figure 2 shows a typical 5 -fold EDPs taken in an as-cast sample. Diffuse streaks are obviously close to the more intense diffraction spots along the 2 -fold diffraction rows. EDPs taken in as cast 

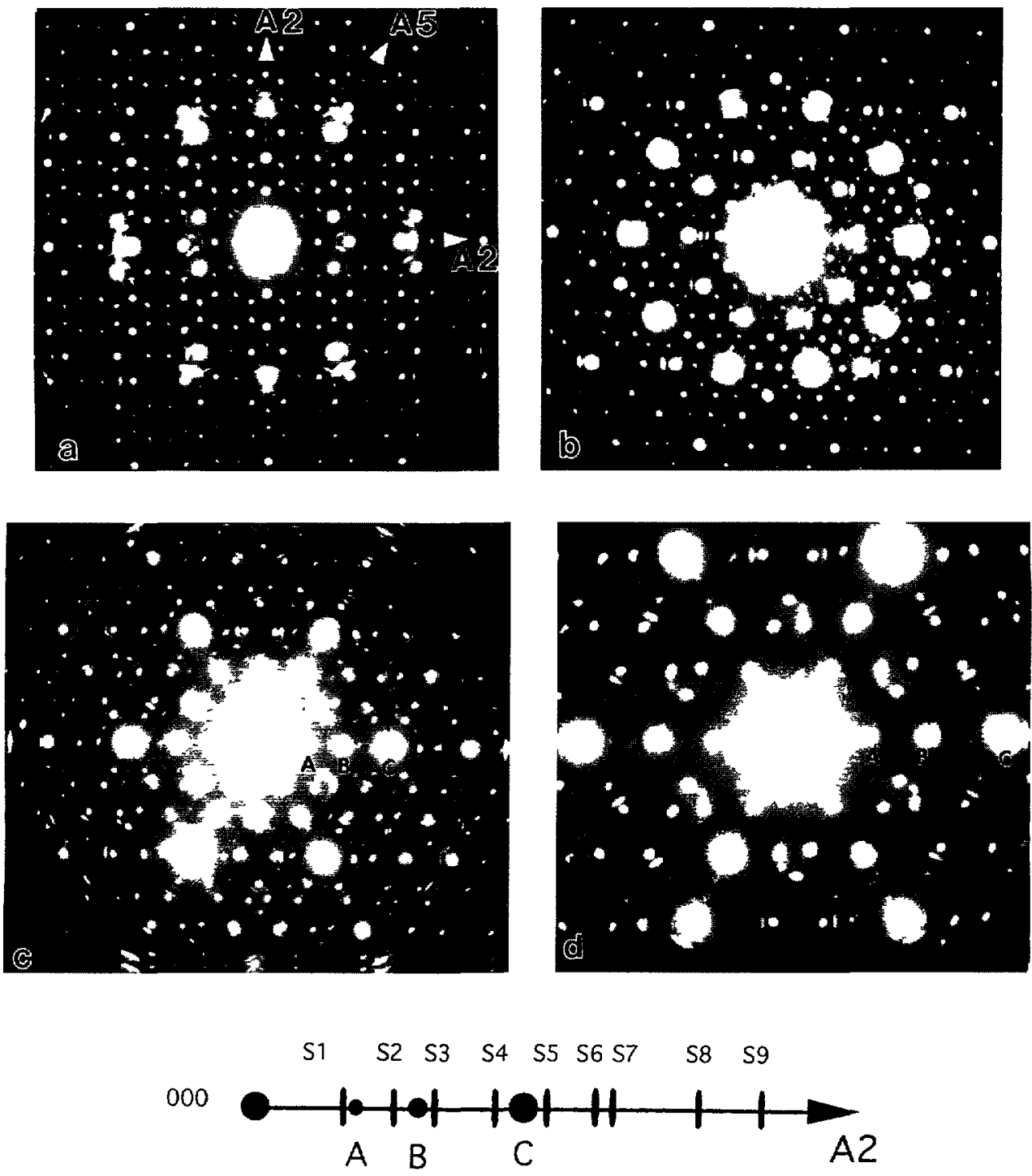

e)

Fig. 1. - Electron Diffraction Patterns (EDPs) from a heat-treated Al-Li-Cu icosahedral phase. The EDP in Figure 1a taken along a 2-fold icosahedral axis exhibits streaks perpendicular to the 2-fold and the 5 -fold diffraction rows (respectively indicated by $\mathrm{A} 2$ and $\mathrm{A} 5$ ). In Figure $1 \mathrm{~b}$ (a diffraction pattern taken along the 5 -fold icosahedral axis), the streaks are found on each of the 2-fold icosahedral diffraction rows in agreement with the observations along the A2 directions in Figure 1a. The EDP 1n Figure 1c has been taken in a transmission electron microscope equipped with an energy filter. Figure $1 \mathrm{c}$ shows that there is a whole famly of streaks along the 2 -fold icosahedral direction. Figure 1d (enlargement of Fig. 1c) reveals the existence of diffuse streaks even at small diffraction angle. The most intense spots along the 2 -fold diffraction row have been labelled A. B, C (their respective 6-d indexations are given in Fig. 4). Figure le sketches the streak posttions along the 2-fold icosahedral axıs. Each streak position is given in Table I (the position of the spot A being taken as the reference). 


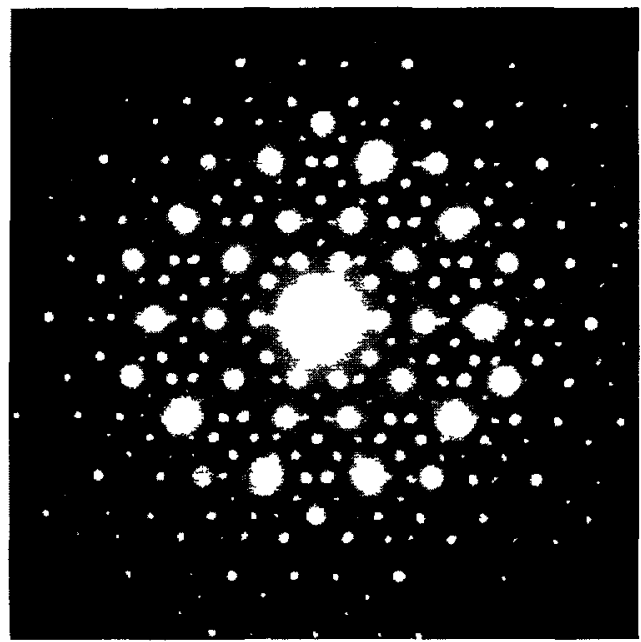

Fig. 2. - Electron diffraction pattern taken along a 5-fold icosahedral axis of an as-cast Al- Li-Cu icosahedral phase. Though less sharp than in Figure 1b, streaks with same direction and position are also present in the as-cast sample.

samples by other authors [11] also show diffuse streaks along the 5-fold diffraction row. Comparing the diffraction patterns of as-cast and heat-treated samples leads then to the conclusion that the same streaks are found in both cases. But the streaks are more intense and sharper in the heat-treated samples. Moreover, the heat-treated samples clearly show that the streaks are always present in many places along the 5 -fold and 2 -fold diffraction rows. Therefore, only the results of the heat-treated samples will be used in the following.

As mentioned above, more or less intense streaks are present in several places on the diffraction patterns. A 2-fold row has been selected in the diffraction pattern from Figure 1c. The diffraction patterns have been digitized and a plot of the scattered intensity along this row has been made to get the streak positions given in Table I. The streak intensities are indicated in the third column of Table I. Obviously, there is no periodicity of the streak positions. It is worth noting that the aperiodicity cannot be attributed to the curvature of the Ewald sphere. Such deviations are only seen at large diffraction angle and generate small deviations (less than several per cent). The diffuse scattering streaks are localized only on one side of the quasicrystal diffraction spots. Some spots actually have streaks on both sides but one is very weak in comparison with the other. This asymmetry indicates that the diffuse streaks are not due to a periodic phenomenon, which would have given diffraction effects symmetrical with respect to the quasilattice spots.

Double diffraction has been suggested to explain the diffuse streaks [11]. Our observation does not support this interpretation since the streaks do not disappear when tilting the sample or the beam. Moreover, the streaks have been observed with different electron wavelengths (Electron microscope operating voltage: $200 \mathrm{keV}$ and $120 \mathrm{keV}$ ). Actually, along a 2-fold icosahedral diffraction row, the diffuse bars occupy always a particular position with respect to the quasicrystal spots. The ratios of the distances between the diffuse bar and that of its neighboured spots and the distance between the two quasicrystal spots surrounding the diffuse bar are given in Table $I$. They are close to $\tau^{-1}$ where $\tau$ is the Golden ratio $(\tau=(1+\sqrt{5}) / 2 \approx 1.618$; $\tau^{-1}=\tau-1 \approx 0.618$ ). This ratio is typical of the quasicrystal diffraction spot positions along the 2-fold icosahedral phase axes. The diffuse streak positions must be indexed with particular sets of indices in the quaslattice in order to exhibit such a ratio. 
Table I. - The ratios of the positions presented in the last column are obtained using intensity plots of digitized diffraction pattern negatives. The streak positions are refered to the position of the spot indexed $(1,0,1,-1,0,1)$ (spot $A$ in Figs. 1c, d,e).

\begin{tabular}{|c|c|c|c|}
\hline Streak label & Streak positions & Observed intensity & Ratio to closest spots \\
\hline S1 & 087 & S & $063 \pm 002$ \\
\hline S2 & 137 & W & $060 \pm 0.02$ \\
\hline S3 & 177 & S & $062 \pm 002$ \\
\hline S4 & 237 & W & $0.64 \pm 002$ \\
\hline S5 & 290 & S & $059 \pm 0.02$ \\
\hline S6 & 3.38 & W & $060 \pm 002$ \\
\hline S7 & 353 & W & $061 \pm 0.02$ \\
\hline S8 & +41 & S & \multirow{2}{*}{ quasicrystal spots } \\
too weak \\
\hline S9 & 503 & S & \multirow{2}{*}{}
\end{tabular}

The 5-fold symmetry diffraction rows show less diffuse scattering streaks: the most prominent ones are located between the diffraction spots indexed by $(28,44)$ and $(18,29)$ using the $N$, $M$ notations [15] (21111- 1 and $31111-1$ in the 6-d lattice). The ratio of the distances of the diffuse streak to each of the neighboring spots is again close to $\tau^{-1}$. The same feature was noticed by Staiger et al. [11]. This typical ratio indicated that the diffuse streaks along the 5 -fold axes are also centered on remarkable positions in the 6-d hyperspace.

Conventional and high resolution images have been made to test whether a second phase was present. Actually we get the usual speckle contrast. It does not seem that the diffuse bars are due to a double diffraction between a microcrystalline layer and the quasicrystal as proposed in [11]. The diffuse scattering streaks on the EDPs were observed regardless of the existence of a Moiré fringe contrast on the images. We have performed the Fourier Transform of high resolution image which appears featureless. We obtained diffraction patterns showing diffuse streaks as also reported by Staiger et al. [11]. This strongly suggests that the diffuse scattering is due to microstructural features.

Figure 3 displays a sequence of diffraction patterns obtained by tilting the sample along a 2 -fold axis (Fig. 3a) or along a 5-fold axis (Fig. 3b). In both cases there is no extinction of the streaks belonging to the tilt axis. It means that the streaks on the EDPs are due to discs of diffuse intensity in the 3-dimensional reciprocal space.

The experimental information suggests two trends for a discussion. One part is to consider the meaning of the reciprocal discs in terms of quasicrystal microstructure, the other relates the aperiodicity of the diffuse discs which suggests to develop a 6-dimensional description.

\section{Basic Concepts for Interpretation}

2.1. Tentative of Description in the 6-Dimension Space. - The correspondence between the observations in the physical space and a 6-dimensional space is not trivial for several reasons. First what is identified as a disc in the 3 d reciprocal space may have a more complex shape in the 6-dimensional space. As a projection is involved, it might even be possible that several solutions provide similar results. In that case, it will be difficult to discriminate between models since electron diffractions give only a rough information on the scattered intensity. Therefore we will focus on the position of the diffuse discs in this 6-d description. 

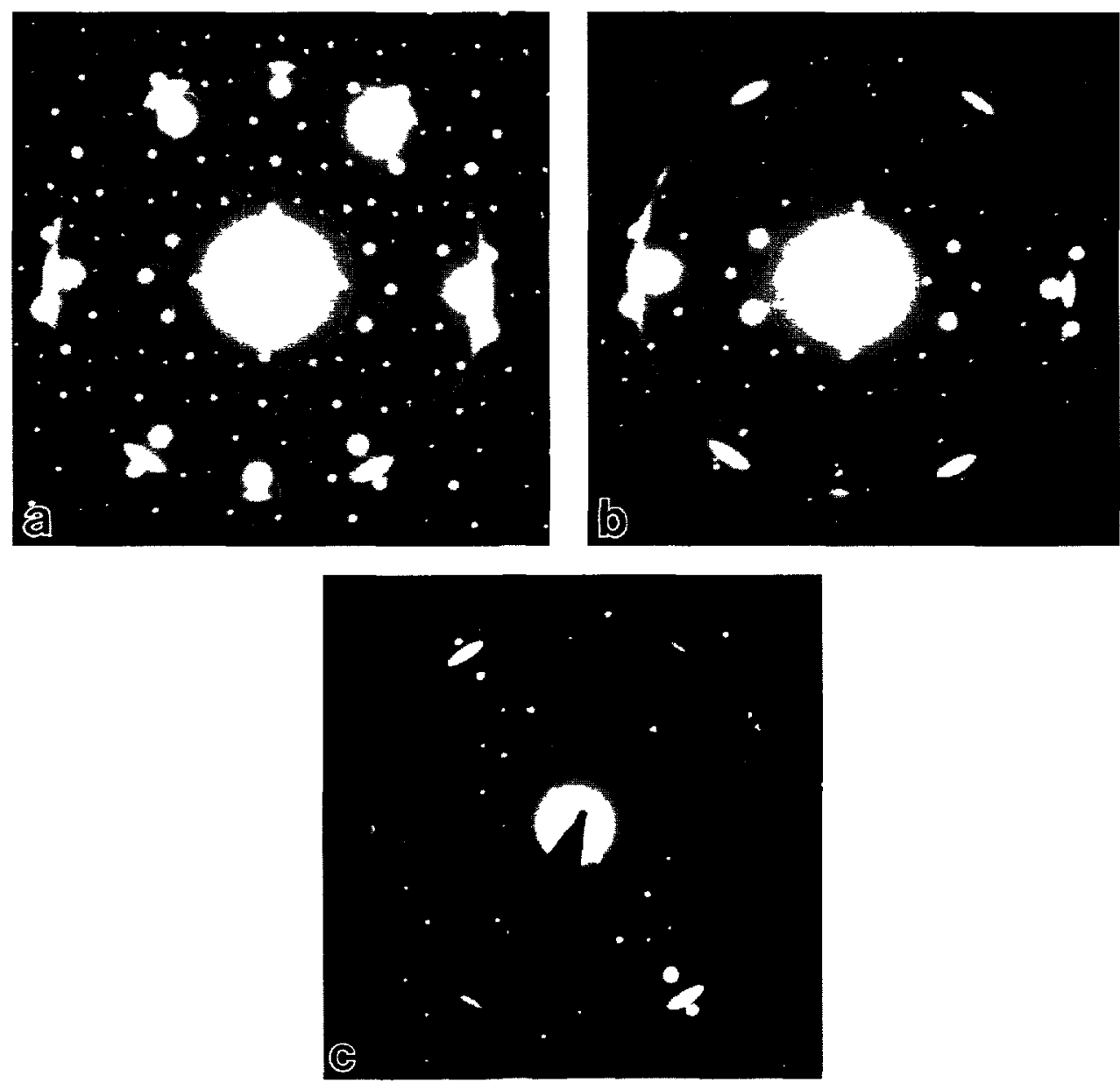

Fig. 3. - a) EDP from a heat-treated Al-Li-Cu icosahedral phase. b) and c): These EDPs are obtained from the 2-fold EDP shown in Figure 3a by tilting either along the 2-fold axis or along the 5-fold axis. In both cases, the streak shape is preserved meaning that the streaks on the EDPs are diffuse discs in the reciprocal space.

A diffraction row in the physical reciprocal space is generated, according to the cut and project algorithm, by a 2-dimensional subspace of the 6-d lattice [16]. As recalled in Figure 4, the 2-fold icosahedral axis is obtained by applynng the cut and project algorithm to a square lattice built on the basis vectors of the 6-d lattice (for a detailed explanation see [16,17]). The distribution of diffraction spots is given by the intersection between the physical space and the reciprocal square lattice convoluted by the Fourler Transform of the selection window. The lattice nodes which are close to the physical space are then the most intense ones. Therefore the intense diffraction spots labelled $\mathrm{A}, \mathrm{B}, \mathrm{C}$ on the expermental diffraction patterns correspond to the lattice nodes indexed by $(1,0,1,-1,0,1),(1.0,2,-1,0,2),(2,3.0,-2,3,0)$. It is easy to report the positions of diffuse scattering given by the experimental electron diffraction patterns: they are indicated by black bars in Figure 4. As mentioned prevously, the Golden proportion observed along the 2 -fold axes indicates that these positions can be indexed as $6-\mathrm{d}$ 


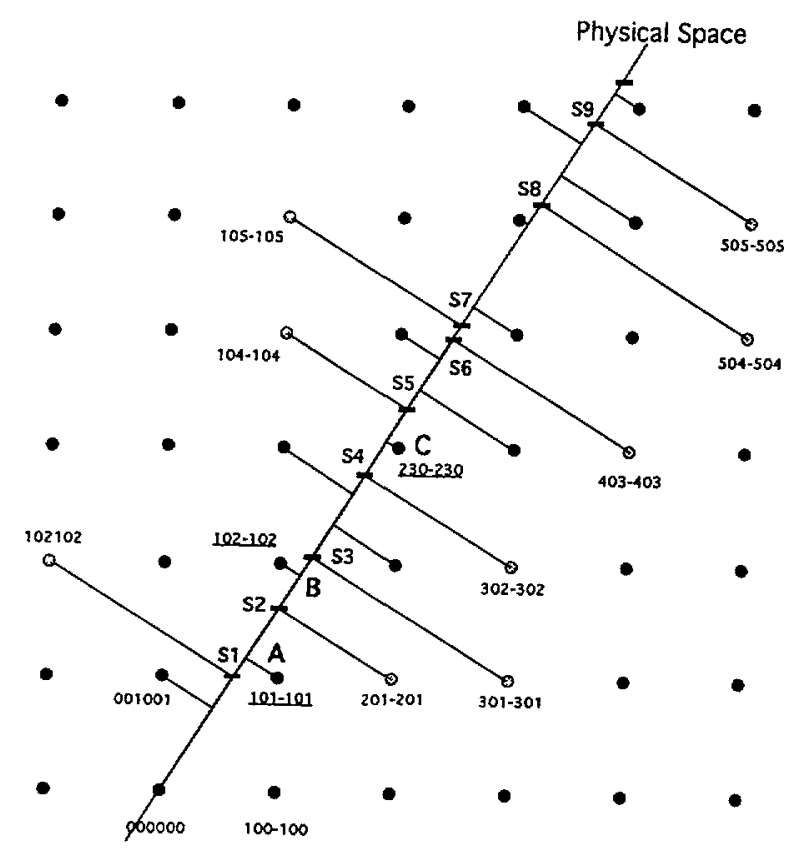

Fig. 4. - Construction of the 2 -fold icosahedral diffraction row according to the Cut-and- Project algorithm. The Fourier Transform of the selection window has been here omitted. The bars labelled S1 to S9 refer to the streaks observed along the 2-fold icosahedral diffraction row (Fig. 1). The 6-d indexation of each streak is easily derived from this construction. To help the comparison with the experimental diffraction patterns (Fig. 1), the lattices nodes giving the spots labelled A, B, C have also been reported with their indexation.

lattice nodes. The streak indexation is obtained by tracing a line from the streak position perpendicularly to the physical space. The lattice nodes intersected by the lines give the indexations of the streaks, they are reported in Table II. The 6-d indexation gives the position as well as the intensity of a spot since the lattice nodes far from the physical space give weaker spots than the nodes close to the physical space. The intensities (weak (W) or strong (S)) expected from the indexation are given in Table II together with the observed intensities. The comparison shows that the quasicrystal indexation accounts very well for the streak positions but clearly not for the intensities.

The cut and project algorithm has been also used to find the indexation of the streaks found along the 5-fold axes. The 5-fold diffraction row is generated by the 6-d canonical vectors: (10000) and $(01111-1)$ as indicated in Figure 5. As above, the observed streak position could be indexed as $1 / 2\left(\begin{array}{llll}3 & 3 & 3 & 3 \\ 3 & -3\end{array}\right)$ which is a node of the $I\left(a^{*}\right)$ reciprocal lattice. This node is quite far from the physical space and, similarly to the streaks along the 2-fold axis, the observed streak intensities cannot be understood within the quasicrystal concept. This disagreement on the intensities reminds what happens in crystals when the intensity is spread out by a size factor. The intensity reveals a reduced peak intensity at the center accompanied by spreading. This suggests that a comprehensive 6-d description of the present diffuse scattermn should involve a 6-d equivalent of a size factor effect. The 6-dimensional crystallography has also proved its validity by the simple relation between observations and 6-d descriptions. For 
Table II. - Comparson between the observed streak intensity and the intensity of quasicrystal spots wath the same indexation.

\begin{tabular}{|c|c|c|c|}
\hline Streak label & Indexation & Intensity after $C P$ & Observed intensity \\
\hline S1 & -102102 & W & S \\
\hline S2 & $201-201$ & S & W \\
\hline S3 & $301-301$ & W & S \\
\hline S4 & $302-302$ & S & W \\
\hline S5 & $104-104$ & S & S \\
\hline S6 & $403-403$ & W & W \\
\hline S7 & $105-105$ & W & W \\
\hline S8 & $504-504$ & W & S \\
\hline S9 & $505-505$ & S & S \\
\hline
\end{tabular}

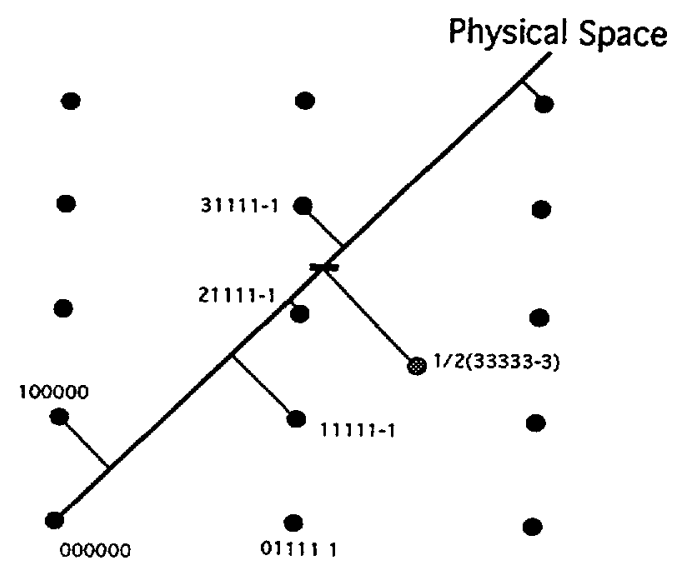

Fig. 5. - Construction of the 5-fold icosahedral diffraction row according to the Cut-and- Project algorithm. The streak position given by the experimental patterns corresponds to a lattice node

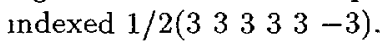

example, what look like dislocations or antiphase boundaries on TEM images of quasicrystals are hyperspace dislocations or antiphase boundaries [18]. Of course, these structural defects do not behave like crystal defects but the analogy works. As we have identified diffuse scattering discs in the physical space, they might correspond to a distribution of 6-d reciprocal space discs enlarged by a size factor.

However, these are only suggestions that can be drawn from the anomalous behavior of the diffuse features; their 6 - $d$ representation remains an open question. In any case, the $6 \mathrm{~d}-$ indexation of the diffuse streaks rules out the icosahedral glass and random tiling models because, in these models, the localized diffuse intensity appears in dark areas meaning that the diffuse rings correspond to positions indexed with high $q_{\text {perp }}$ components. On the contrary, the indexation of the diffuse streaks gives moderate $q_{\text {perp }}$ components. In addition, the streak intensities are not in agreement with their respective $q_{\text {perp }}$ components. As the models usually 
explaining the diffuse rings appear to be irrelevant for the diffuse streaks, crystallographic 6-d models should be considered. Actually, before building 6-d models, it should be interesting to interpret, first, the observations in the 3 -d space. Such interpretation may be useful for models based on analogy between 6-d and 3-d space.

2.2. A Microstructural interpretation of Diffuse Scattering Discs. - We now consider the meaning of diffuse intensity discs in the 3 -d reciprocal space. Usually diffuse intensities are essentially due to a disorder phenomenon or a shape factor. For instance, diffuse scatterings are frequently met during a short range order/long range order (SRO/LRO) transition. In that case, the diffuse intensities are observed along directions concerned by the ordering/disordering process and are centered on the extinguished spots due to the disordered phase. Such a transition has been observed in the approximant AlMnFeSi-Alpha phase where the diffuse scattering forms more or less continuous shells [19]. A SRO/LRO interpretation is proposed by Mukhopadhay et al. [5] for the diffuse scattering arcs in Al-Mn quasicrystalline phases. Obviously, the present diffuse intensity discs do not seem to belong to such an ordering effect: they are actually too well defined in comparison with the diffuse shapes generated by this type of disorder.

One possible interpretation for the streaks consists in noting that, because of the Fourier Transform relating the direct and reciprocal space, a disc in the reciprocal space is associated to a small elongated object in the direct space. It means that the sample microstructure could contain small elongated needles or stringlets. As mentioned previously the HREM images are featureless. This is not surprising since the size of the diffuse discs indicates very small needles: several nanometer long. It should be mentioned that needles have only been observed on samples annealed for 72 hours at $575^{\circ} \mathrm{C}$ [14]. Possibly, the annealing has allowed the growth of stringlets into observable needles. The question is then which species are involved in these stringlets. The quasicrystal structure being built by a packing of oblate and prolate rhombohedra suggest that some rhombohedral units might show defects either because of vacancies or peculiar atomic decoration. The packing of faulted rhombohedral units is in good agreement with our observations for the following reasons. First piling several oblate or prolate faulted rhombohedra build a stringlet of about 1 nanometer. Second, the rhombohedra facets being normal either to 2-fold axes or to 5 -fold axes, their packing will generate diffuse discs having their axis parallel to 2 -fold or 5 -fold icosahedron axes.

This explanation in terms of stringlets of faulted rhombohedral units is quite at tractive since it allows a connection between rings and discs of diffuse intensity. The rings might correspond to defective units scattered over the sample while the diffuse discs are generated by pling up defective units. An open question is the nature of such faulted rhombohedra. The pling of defect units is reminiscent of the crystal vacancy behavior that tend to form divacancies, trivacancies under annealing. In other respect, numerous electron phases ( $\gamma$-brass, CoAl, NiAl) provide examples of strong correlations between vacancies and the structure of Hume-Rothery phases [20]. It is then reasonable to think that the quasicrystal structure may contain a quite large density of vacancies without alteration of the quasicrystal structure.

\section{Conclusion}

The observations reported here confirm that diffuse scattering is a rich and original feature of quasicrystalline phases. The diffuse scatterıng streaks exhibited by the Al-Li-Cu icosahedral phase are analyzed and are shown to be diffuse discs in the 3-d reciprocal space. We confirm what was already observed in other icosahedral quasicrystals: either diffuse discs and rings are centered on well-defined positions indexable with the 6-dimensional crystallography. Still, an 
adaptation of the 6-d description is required to account for the diffuse discs. However, it is possible to interpret the discs of diffuse scattering within the 3-d direct space. Stringlets made by several faulted rhombohedral units plled face-to-face agree with the characteristics of the diffuse effects observed in the Al-Li-Cu icosahedral phase (size, shape, directions). This 3-d interpretation is given for two reasons. First, it might be a basis for a 6 -d model build on the analogy between $3-d$ and $6-d$ descriptions. The second point is that this interpretation emphasizes on the presence of vacancies. These defects are rarely considered in quasicrystal studies, though vacancies may become a very important point, in particular because of the increasing interest for atomic diffusion. It is worth noting that, though the diffuse scatterings have been studied on specifically heat-treated samples, the same effects, but less pronounced, are met in most of the $\mathrm{Al}-\mathrm{Li}-\mathrm{Cu}$ icosahedral phase samples. Another possibility for the interpretation would be then to consider that the scattering discs are related to the Hume-Rothery mechanism responsible for the stability of the Al-based quasicrystal. In that case, a comparison between the distribution of the most intense streaks and the Fermi surface should be fruitful.

\section{Acknowledgments}

The authors are grateful to Drs. Sainfort and Dubost from the Péchiney Research Centre for providing the as-cast quasicrystalline sample. The C.I.M.E is acknowledged for providing the Zeiss EM 912 transmission electron microscope facilities.

\section{References}

[1] Henley C.L., Quasicrystals, the state of the art, Directions in Condensed Matter, Vol. 11, D.P. Di Vincenzo and P.J. Steinhardt Eds. (1988) pp. 429-524.

[2] Menguy N., de Boissieu M., Guyot P., Audier M., Elkaim E. and Laurat J.P., J. Non-Cryst. Sol. 153-154 (1993) 620.

[3] Dénoyer F., Heger G., Lambert M., Lang J.M. and Sainfort P., J. Phys. France 48 (1987) 13571361 .

[4] Goldmann A.I., Guryan C.A., Stephens P.W., Parsey J.M., Aepplı G., Chen H.S. and Gayle F.W., Phys. Rev. Lett. 61 (1988) 17, 1962-1965.

[5] Mukhopadhay N.K., Ranganathan S. and Chattopadhyay Phıl. Mag. Lett. 56 (1987) 4, 121.

[6] Gibbons P.C., Kelton K.F., Levine L.E. and Phillıps R.B., Phıl. Mag. B. 59 (1989) 593-618.

[7] Zhang X. and Kelton K.F., Phal. Mag. Lett.,4 62 (1990) 265-271.

[8] Newman M.E.J., Henley C.L. and Oxborrow M., Phul. Mag. B, 571 (1995) 991-1013.

[9] Gibbons P.C. and Kelton K.F., J. Non-Cryst. Sol. 153-154 (1993) 165-170.

[10] Staiger W., Yu D.P. and Kléman M., Phıl. Mag. A., 467 (1993) 991-1005.

[11] Yu N., Portier R., Yu-Zhang K., and Bigot J., Phul. Mag. Lett. 57 (1988) 1, 35-39.

[12] Kim D.H., Hutchison J.L. and Cantor B., Phıl. Mag. A. 61 (1990) 2, 167-192.

[13] Sainfort P. and Dubost B., J. Phys. Colloq. France 47 (1986) C3-321.

[14] Donnadieu P., Wang K., Degand C. and Garoche P., J. Non-Cryst. Sol. 183 (1995) 100-108.

[15] Cahn J.W., Shechtman D. and Gratias D., J. Mater. Res. 1 (1986) 1, 13.

[16] Duneau M. and Katz A., Phys. Rev. Lett. 54 (1985) 2688.

[17] Janot C., Quasicrystals, a primer, Monographs on the physics and chemistry of materials, Vol 48 (Oxford Science Publications, 1992).

[18] Devaud-Rzepsky J., Quivy A., Calvayrac Y., Corner-Quiquandon M. and Gratias D., Phılos. Mag. B 60 (1989) 6, 855 .

[19] Donnadieu P., Lapasset G. and Sanders T.H., Phılos. Mag. Lett. 70 (1994) 5, 319-326.

[20] Cahn R.W., Nature 279 (1979) 579-581. 